\title{
Ultrashort Long-Period Fiber Gratings Inscribed on a Single-Mode Fiber for Torsion Sensing Applications
}

\author{
Marta Nespereira $\mathbb{D}^{1}{ }^{1}$ João M. P. Coelho $\mathbb{D}^{1,2}$ and José Manuel Rebordão $\mathbb{D}^{1}$ \\ ${ }^{1}$ Laboratório de Óptica, Lasers e Sistemas, Faculdade de Ciências, Universidade de Lisboa, 1649-038 Lisboa, Portugal \\ ${ }^{2}$ Instituto de Biofísica e Engenharia Biomédica, Faculdade de Ciências, Universidade de Lisboa, 1749-016 Lisboa, Portugal \\ Correspondence should be addressed to João M. P. Coelho; joao.coelho@fc.ul.pt
}

Received 28 June 2018; Revised 9 November 2018; Accepted 14 November 2018; Published 6 December 2018

Academic Editor: Wojtek J. Bock

Copyright (c) 2018 Marta Nespereira et al. This is an open access article distributed under the Creative Commons Attribution License, which permits unrestricted use, distribution, and reproduction in any medium, provided the original work is properly cited.

\begin{abstract}
The response of ultrashort-length $\mathrm{CO}_{2}$-induced long-period fiber grating (LPFG) sensors to torsion is reported. While engraving using $\mathrm{CO}_{2}$ laser radiation, the fiber is submitted to high tension allowing the obtainment of gratings with shorter lengths, down to $2.4 \mathrm{~mm}$, which is almost one order of magnitude lower than the usual. Also, the fiber is only irradiated in one side, creating an asymmetrical profile leading to highly birefringent gratings. Sensitivity to axial twists is demonstrated, with values up to $0.15 \mathrm{~nm} /(\mathrm{rad} / \mathrm{m})$ for the resonant wavelength shift and higher than $0.03 \mathrm{dBm} /(\mathrm{rad} / \mathrm{m})$ for the variation in the intensity (attenuation). Discrimination between rotation directions, clockwise and counterclockwise, was observed.
\end{abstract}

\section{Introduction}

In recent decades, the use of optical fiber-based sensors has been increasing due to the advantages of this type of structures: high sensitivity, compact size, and immunity to electromagnetic interference. For civil engineering, twist displacement (rotation) is an important parameter for monitoring the structural health of buildings, bridges, or dams; in the field of robotics, the potential applications are easily envisaged.

Different fiber-based sensing structures have been developed for torsion detection, such as long-period fiber gratings (LPFGs) based on screw-type distortion in the bare optical fiber [1], the engraving of angle-chirped LPFGs [2], helicoidal long-period fiber gratings (H-LPFGs) [3], twist tapers, and special fibers like the polarization-maintaining fibers (PANDA, Bow Tie) for their high linear birefringence [4]. Other approaches include interferometric configurations (Sagnac, Mach Zehnder) and loop mirrors, resulting in more complex structures.

In this work, the response of long-period fiber gratings (LPFGs) manufactured by $\mathrm{CO}_{2}$ laser radiation to torsion in an asymmetrical fiber exposure configuration is discussed.
First presented (for this application) by the authors in [5], such gratings have short length and slightly taperized structures, due to the high tension the fiber is submitted to during manufacture, leading to highly birefringent structures within the fiber's cross section. The theoretical principles are detailed, and their analysis is now extended and their sensing ability is further tested by monitoring the changes in the resonant wavelength peak and transmitted light intensity when the fiber is twisted.

For comparison purposes, Table 1 includes different values of torsion responses from the literature and the range of sensitivities from $\sim 0.02$ to $0.22 \mathrm{~nm} /(\mathrm{rad} / \mathrm{m})$ for simple LPFG configurations to $\sim 0.593$ to $2.392 \mathrm{~nm} /(\mathrm{rad} / \mathrm{m})$ for more complex ones. The sensitivity is expressed as a function of the torsion rate (in $\mathrm{rad} / \mathrm{m}$ ) because it depends on the total length of the fiber being twisted. It is important to mention that in all cases, the sensor can discriminate between torsions' sense.

The reported grating lengths have values between $\sim 10 \mathrm{~mm}$ and $40 \mathrm{~mm}$, corresponding to a number of grating planes from 20 to 45 , depending on the value of the period. Although in principle there is no direct correlation between grating length (or number of grating planes) and sensitivity performances, it can be advantageous to have the same 
TABLE 1: Values of sensitivity found in literature for torsion measurement for simple LPFGs or other LPFG-based configurations.

\begin{tabular}{lcc}
\hline Reference & $\begin{array}{c}\text { Sensitivity } \\
(\mathrm{nm} /(\mathrm{rad} / \mathrm{m}))\end{array}$ & $\begin{array}{c}\text { Grating length } \\
(\mathrm{mm})\end{array}$ \\
\hline Deng et al. [1] & 0.1604 & 9.84 \\
Li et al. [2] & $\sim 0.593-\sim 0.94$ & $*$ \\
Zhang et al. [3] & 0.115 & 17.1 \\
Zhou et al. [4] & $1.071-2.392$ & $*$ \\
Rao et al. [9] & 0.2244 & 40 \\
Wang and Rao [10] & $\sim 0.0645$ & 20 \\
Rao et al. [11] & 0.0645 & 19.6 \\
Wang and Rao [12] & 0.024 & 27 \\
Shi et al. [13] & 0.084 & $*$ \\
Dong et al. [14] & $0.1187-0.1817$ & $*$ \\
\hline
\end{tabular}

${ }^{*}$ Not specified.

torsion response with a smaller number of grating planes, in order to induce less damage to the fiber. In addition, a smaller grating length can facilitate the creation of more complex structures, like multiplexed or coated LPFGs. However, engraving shorter-length gratings has specific practical challenges since typical procedures cannot be followed [6].

Although the sensitivity of this type of grating can be enhanced through mirroring and film depositions [7, 8], the present work does not include these techniques.

\section{Theoretical Principles}

LPFGs are periodic structures engraved in the optical fiber along its longitudinal axis, with the purpose of inducing a slight change in the refractive index of the fiber core. Their working principle is explained by coupled-mode theory [15], which describes how part of the light of the fundamental guided mode (core mode) of the optical fiber is coupled to one or more of the copropagating cladding modes when it propagates through a LPFG, as well as power transfer between the core and cladding modes.

These devices are characterized by their fabrication parameters: period, length, and the induced refractive index variation between core and cladding. The phase matching condition shows the relation between these parameters and is given by [15].

$\mathrm{CO}_{2}$ laser radiation-engraved gratings exhibit special features that allow the fabrication with very short lengths and explain their torsion sensitivity and torsion sense discrimination. According to [16], the effective refractive index modulation is achieved by physical mechanisms connected to the residual stress generated in the fiber during the engraving process, which is derived from the different thermal expansion coefficient and viscoelastic properties in the core and cladding, changes in the glass structure (volume increase and densification), and, finally, physical deformation such as elongations or taper effect.

These mechanisms are combined with the high tensile strength the fiber was submitted to by pulling it with a mass while irradiating. It is well known that the coupling coefficient $\kappa$ depends on the mechanical tension applied to the fiber [6]; the higher the tension, the higher the $\kappa$. Also, having that in mind, in LPFG, one can show that $\kappa$ is proportional to the refractive index modulation, and it can be assumed that the induced changes in the refractive indexes are also high. This is in accordance with the expression for $\kappa$ : if the grating's length $\left(L_{\text {grating }}\right)$ is very small, $\kappa$ must be high:

$$
\kappa L_{\text {grating }}=\left(\frac{\pi}{2}\right) \kappa L_{\text {grating }}=\frac{\pi}{2} .
$$

Other significant feature of $\mathrm{CO}_{2}$ laser-induced gratings (at $10.6 \mu \mathrm{m}$ ) is the asymmetrical exposure of the fiber (it is irradiated from one side), inducing uneven radial and azimuthal variations in the refractive index of the fiber materials. It is, therefore, considered that $\mathrm{CO}_{2}$ laser-induced gratings are highly birefringent along the fiber's axis. As demonstrated in the previous work [6], the coupled-mode theory and 3D finite element modeling for predicting the refractive index variation produced by the laser irradiation are adequate to model and understand the mechanisms enabling the creation of ultrashort LPFGs. In fact, both theoretically and experimentally, it was demonstrated that the amount of strain applied to the fiber plays an important role in the quality of the sensors and has a direct relation to the required lengths of the gratings. Typically, higher pulling forces allow shorter LPFGs, while lower strains lead to larger lengths. In the first case, the strain increases the tapering effect and can produce breakage. In the second case, as the pulling force is reduced, a larger number of periods are necessary in order to create useful sensing devices with significant attenuation on the resonant modes [6].

The sensitivity of LPFGs to torsion arises from the connection between changes in the grating's birefringent structure due to axial twists (caused by the photoelastic effect) and the state of polarization (SOP) of the guided light $[10,16,17]$, producing a shift of the resonance wavelength and/or an increment or reduction of the intensity of the transmitted light.

According to coupled-mode theory, cladding modes are characterized by their effective refraction index and propagation constant. For each SOP, there are specific resonances determined by an effective refraction index of the core and the cladding modes. The variation in the effective refraction index of the cladding modes determines the shift in resonance wavelengths [17]. Bearing that in mind, for $\mathrm{CO}_{2}$ laser-induced LPFGs, one can show that birefringence is only induced in the cladding, and the wavelength shift is given by

$$
\Delta \lambda_{\text {res }} \approx \Lambda\left(n_{l m}^{\text {eff,max }}-n_{l m}^{\text {eff,min }}\right)
$$

where $n_{l m}^{\text {eff,max }}$ and $n_{l m}^{\text {eff,min }}$ are the maximum and minimum effective refractive index for the $\mathrm{LP}_{l m}$ cladding mode, respectively, over all polarization states [17].

When applying torsion, the change of the SOP can lead to any state of polarization, from linear to elliptical $[10,16]$, being proportional to the twist rate (twist angle per unit of 


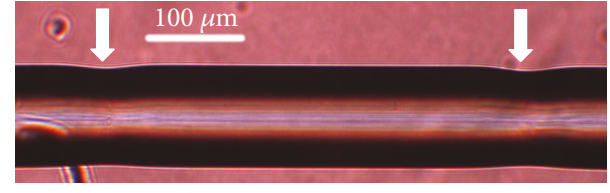

FIgURE 1: Micrograph showing the tapering effect (arrows) on the irradiated zones in a LPFG with $600 \mu \mathrm{m}$ period written on an SMF-28 optical fiber. $\left(P \approx 4.5 \mathrm{~W} ; t_{\text {on }}=0.6 \mathrm{~s} ; F \approx 0.78 \mathrm{~N}\right)$.

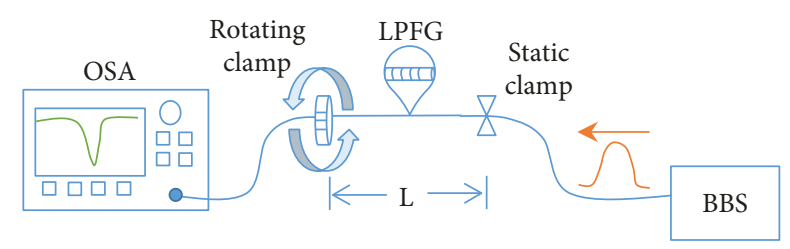

FIGURE 2: Schematic of the setup used for torsion sensing tests.

twisted length). The sense of the elliptical polarization rotation (left or right) is determined by twist direction $[10,16]$. The capability to discriminate twist direction is an important advantage of LPFGs in comparison to interferometric and standard polarization maintaining fibers (PANDA, Bow Tie).

\section{Experimental Methodologies and Results and Analysis}

LPFGs are obtained by periodically sweeping the bare fiber with a $\mathrm{CO}_{2}$ laser beam (Synrad, $25 \mathrm{~W}$ ) focused with a cylindrical lens (working distance of $50 \mathrm{~mm}$ ) into a vertical line of $3 \mathrm{~mm}$ length and $125 \mu \mathrm{m}$ wide. The process is controlled using an automatic system programmed in LabView@ that coordinates the translation stage with the fiber's exposure time and regulates the average laser power, $P$. The detailed manufacturing procedure is explained in Coelho et al. [18] and Nespereira et al. [19].

A set of LPFGs were fabricated with a $600 \mu \mathrm{m}$ period and lengths varying between $2.4 \mathrm{~mm}$ and $7.2 \mathrm{~mm}$. In this section, some results are presented illustrating our study. A weight of around $80 \mathrm{~g}$ (corresponding to a pulling force of $0.78 \mathrm{~N}$ ) was applied to the engraving fibers, therefore inducing a high strain into the fibers. In Figure 1, a microphotograph of a grating is presented, highlighting the tapered regions (notches) produced by the laser irradiation $(P \approx 4.5 \mathrm{~W}$ for a duration of $t_{\mathrm{on}} \approx 0.6 \mathrm{~s}$ ), a typical result of using this type of process.

Then, torsion tests were performed by using a special clamp to control rotation (Thorlabs HFR007, fiber rotator for a bare fiber with a $125 \mu \mathrm{m}$ cladding diameter). Figure 2 illustrates the methodology.

After tensing the grating, a static clamp fixes the fiber tightly, allowing twisting the grating clockwise or anticlockwise, while an optical spectrum analyzer (OSA, Agilent 86140B) records the spectrum for each angle while illuminating the sensor by a broadband source, a super luminescent diode (Thorlabs S5FC1005S), with a central wavelength of $1550 \mathrm{~nm}$ and a spectral bandwidth of $\sim 160 \mathrm{~nm}$. The twist rate is calculated by dividing the torsion angle (expressed in radian) by the distance, $L$, between the fixed end and the rotation point (in our case, $L=0.125 \mathrm{~m}$ ).

The plot in Figure 3 shows the shifts in a spectrum of a grating with $7.2 \mathrm{~mm}$ length and $600 \mu \mathrm{m}$ period, when torsion is applied. The torsion range was $0^{\circ}$ to $360^{\circ}$, a twist rate of $0-50 \mathrm{rad} / \mathrm{m}$. When the grating is twisted clockwise, the spectrum shifts towards shorter wavelengths (blue shift) and the intensity of the resonance peak becomes less pronounced. In the case of counterclockwise twists, the opposite occurs: the resonance wavelength increases (red shift) and the resonance peak becomes more pronounced. The grating is therefore able to distinguish torsion directions while being sensitive to both parameters, wavelength and attenuation. This seems to be in concordance with the hypothesis described in Section 2 and by several authors [10, 16, 17].

For each of the variables (resonance wavelength, attenuation, and twist rate), calibration can be performed in order to have a first approach to their sensing abilities. Figure 4(a) shows the least squares method linear regression of the resonance wavelength $\left(\lambda_{\text {resonance }}\right)$ when twisting clockwise and anticlockwise. We obtained a sensitivity of $0.12 \mathrm{~nm} /$ $(\mathrm{rad} / \mathrm{m})$ for a $7.2 \mathrm{~mm}$ length grating and a sensitivity of $0.09 \mathrm{~nm} /(\mathrm{rad} / \mathrm{m})$ for the shorter $2.4 \mathrm{~mm}$ grating. The calibration of the attenuation response was also made. Figure 4(b) shows the resulting linear regression, showing a sensitivity of $0.03 \mathrm{dBm} /(\mathrm{rad} / \mathrm{m})$ for the $7.2 \mathrm{~mm}$ grating and a sensitivity of $0.06 \mathrm{dBm} /(\mathrm{rad} / \mathrm{m})$ for the $2.4 \mathrm{~mm}$ grating.

In Figure 5, we compare results for LPFGs with different lengths, in the interval from $2.4 \mathrm{~mm}$ to $7.2 \mathrm{~mm}$, and sensitivities for the resonance shift are of the same order of magnitude. The values lie between 0.15 and $0.10 \mathrm{~nm} /(\mathrm{rad} / \mathrm{m})$ for the gratings within this interval. Two types of repeatability were studied: one regarding the capability of obtaining the same performance with different devices with the same grating period and length and the other regarding testing the same sensor on different torsion cycles. The first repeatability is illustrated in Figure 5 through the error bars presented in the plot. This uncertainty is consistent with the intrinsic manufacturing repeatability, as previously studied by the authors [19]. In the second case, we found that the sensitivity values of each sensor for different torsion cycles remained within a variation of less than $2 \%$ (in average, $1 \%)$. Nevertheless, the future evolution of these sensors will involve a more extensive metrological characterization [20] and a more detailed repeatability study.

No similar analysis is presented regarding the attenuation shift because sensitivity usually lies within $0.08-0.03 \mathrm{dBm} /$ $(\mathrm{rad} / \mathrm{m})$ or lower, demonstrating that using this feature is still not reliable.

In addition to twisting the fiber sensors between $0^{\circ}$ and $360^{\circ}$ (equivalent to twisting 8 turns in the fiber), we also analyzed larger twisting ranges, in particular $540^{\circ}$ (one turn and a half) or $720^{\circ}$ (two complete turns). We illustrate in Figure 6 the response for a twist range of two turns for clockwise and counterclockwise sense for a grating with a period of $600 \mu \mathrm{m}$ and $2.4 \mathrm{~mm}$ length. Although sensitivity values are similar to those obtained for smaller twist ranges, their responses are less linear and, often, the grating breaks. 


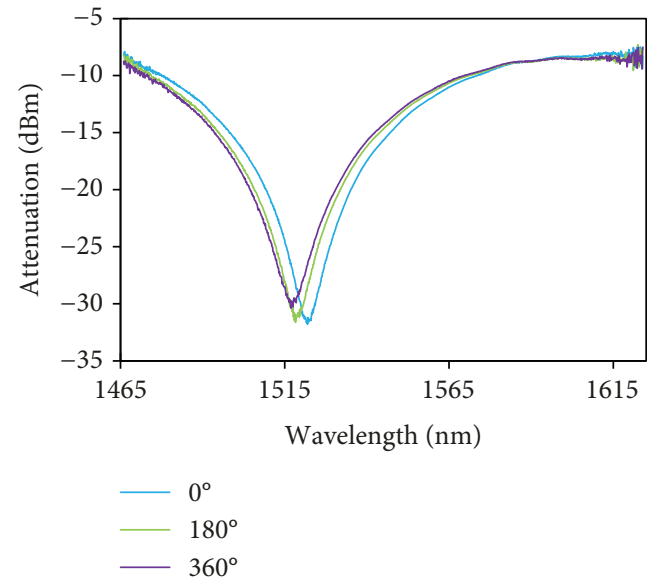

(a)

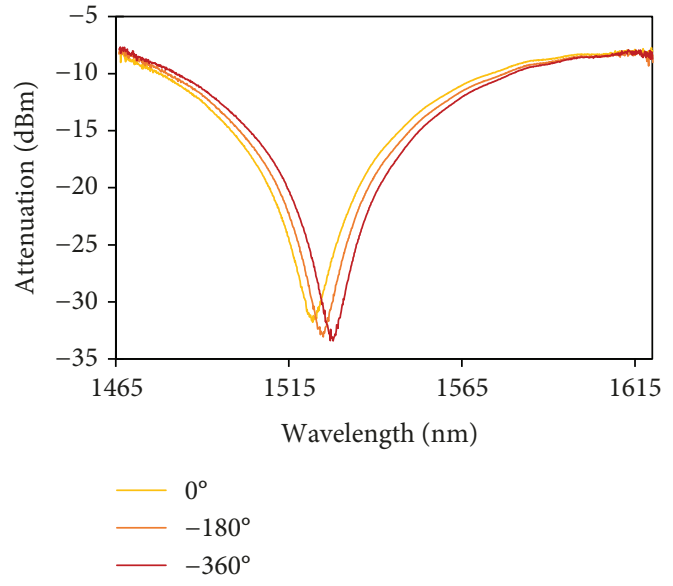

(b)

FiguRE 3: Spectrum shift for a (a) clockwise and (b) counterclockwise axial torsion of a grating with $7.2 \mathrm{~mm}$ length and $600 \mu \mathrm{m}$ period.

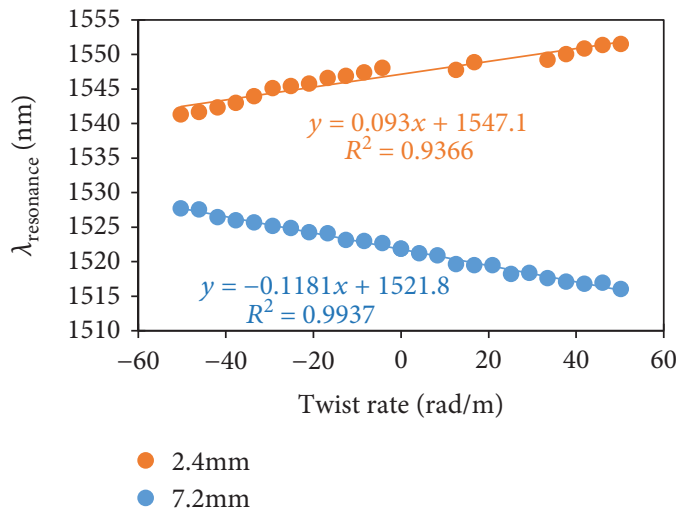

(a)

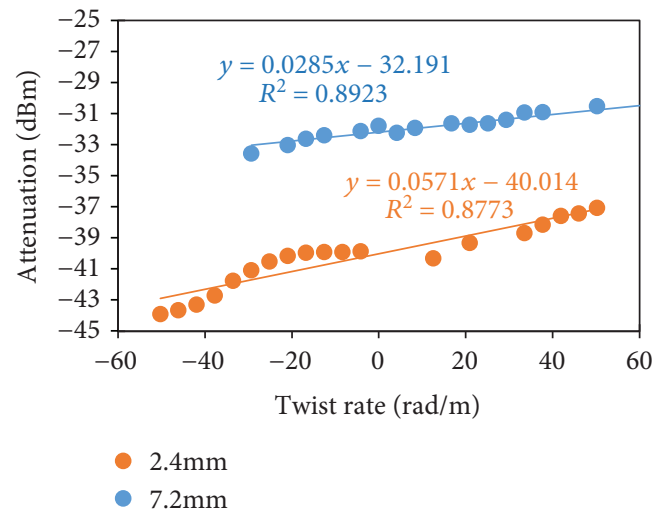

(b)

FIGURE 4: LPFG sensor linear calibration of the (a) resonance and (b) attenuation responses for clockwise and counterclockwise axial torsion, for two LPFG lengths: $2.4 \mathrm{~mm}$ and $7.2 \mathrm{~mm}$.

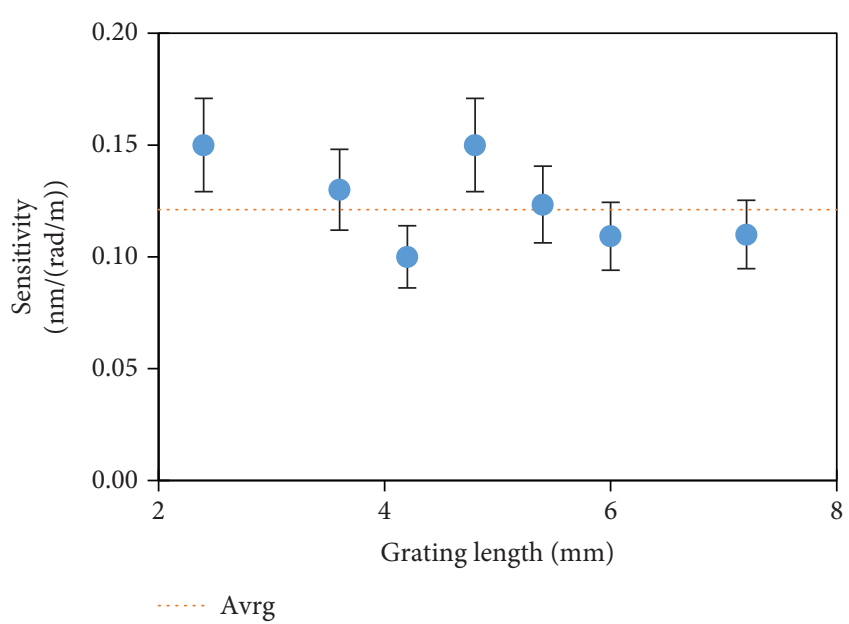

FIGURE 5: Comparison of sensitivity for a set of LPFGs with lengths between 2.4 and $7.2 \mathrm{~mm}$ and a period of $600 \mu \mathrm{m}$, for both clockwise and counterclockwise axial torsion. The pointed line represents the average value, at $0.12 \mathrm{~nm} /(\mathrm{rad} / \mathrm{m}) \pm 0.03 \mathrm{~nm} /(\mathrm{rad} / \mathrm{m})$, and the error bars indicate the uncertainty on sensitivity for different sensors with similar gratings.

\section{Conclusions}

An efficient method for fabrication of short-length and highly birefringent LPFGs is presented, demonstrating its effectiveness for torsion sensing applications. The tested gratings show a response when submitted to axial twists, with sensitivities higher than $0.03 \mathrm{dBm} /(\mathrm{rad} / \mathrm{m})$ for the attenuation shift and up to $0.15 \mathrm{~nm} /(\mathrm{rad} / \mathrm{m})$ for the resonance shift. For the range of lengths being studied (between $2.4 \mathrm{~mm}$ and $7.2 \mathrm{~mm}$ ), the resonance shift has better performance and reliability, being a good candidate for a sensing device. The measurement range was from $0^{\circ}$ to $360^{\circ}$, and the gratings were fabricated with a $600 \mu \mathrm{m}$ period.

Shorter gratings presented a slightly tapered structure which increases mode coupling, leading to higher sensitivity to mechanical deformations. The presence of high birefringence in the fiber glass is assumed, and it should be studied in more depth. In general, better understanding of the behavior of tapered-grating structures is a challenging research topic for advancing in this field.

Also, the creation of more complex sensing devices, such as concatenated LPFG or thin film coating, multiplexing 


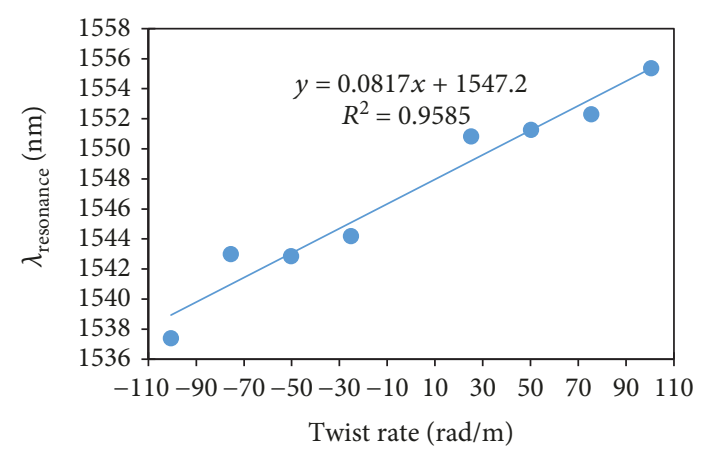

(a)

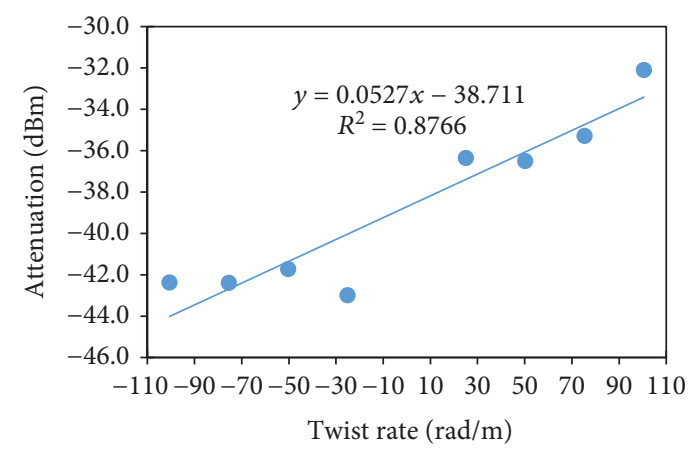

(b)

Figure 6: Example of the response in (a) resonance and (b) attenuation of a $2.4 \mathrm{~mm}$ grating for a torsion of $720^{\circ}$ (two turns) in both clockwise and counterclockwise senses.

LPFG, and mirror coating as well as interferometric sensors aspiring to emulate the state of the art of the subject can be considered for future work.

These specific ultrashort-length LPFG sensors can be useful in situations where a precise measurement of torque is needed. For example, in robotic arms for microsurgery or systems that imitate the movements of the human body, the grating's reduced size can be advantageous. This will also be the object of research in the future.

\section{Data Availability}

The experimental, theoretical and bibliographic data used to support the findings of this study are included within the article.

\section{Conflicts of Interest}

The authors declare that they have no conflicts of interest.

\section{Acknowledgments}

The authors would like to thank Fundação para a Ciência e Tecnologia (FCT) for the financial support under the project UID/BIO/00645/2013. The authors gratefully thank Catarina Silva, David Castro Alves, Fernando Monteiro, and António Oliveira for their support.

\section{References}

[1] M. Deng, J. Xu, Z. Zhang et al., "Long period fiber grating based on periodically screw-type distortions for torsion sensing," Optics Express, vol. 25, no. 13, pp. 14308-14316, 2017.

[2] Y.-P. Li, L. Chen, Y. X. Zhang et al., "Realizing torsion detection using berry phase in an angle-chirped long-period fiber grating," Optics Express, vol. 25, no. 12, pp. 13448-13454, 2017.

[3] H.-L. Zhang, W. G. Zhang, L. Chen et al., "Bidirectional torsion sensor based on a pair of helical long-period fiber gratings," IEEE Photonics Technology Letters, vol. 28, no. 15, pp. 1700-1702, 2016.

[4] Q. Zhou, W. Zhang, L. Chen et al., "Fiber torsion sensor based on a twist taper in polarization-maintaining fiber," Optics Express, vol. 23, no. 18, pp. 23877-23886, 2015.

[5] M. Nespereira, J. M. Coelho, and J. M. Rebordão, "Shortlength long period fiber grating for torsion sensing applications," in Proceedings Volume 10453, Third International Conference on Applications of Optics and Photonics; 104531Z, Faro, Portugal, August 2017.

[6] M. Nespereira, J. M. P. Coelho, M. Abreu, and J. M. Rebordão, "Ultrashort long-period fiber grating sensors inscribed on a single mode fiber using $\mathrm{CO}_{2}$ laser radiation," Journal of Sensors, vol. 2017, Article ID 4196431, 9 pages, 2017.

[7] A. Singh, "Long period fiber grating based refractive index sensor with enhanced sensitivity using Michelson interferometric arrangement," Photonic Sensors, vol. 5, no. 2, pp. 172-179, 2015.

[8] L. Coelho, D. Viegas, J. L. Santos, and J. M. M. M. De Almeida, "Characterization of zinc oxide coated optical fiber long period gratings with improved refractive index sensing properties," Sensors and Actuators B: Chemical, vol. 223, pp. 45-51, 2016.

[9] Y. J. Rao, T. Zhu, and Q. J. Mo, "Highly sensitive fiber-optic torsion sensor based on an ultra-long-period fiber grating," Optics Communication, vol. 266, no. 1, pp. 187-190, 2006.

[10] Y. P. Wang and Y. J. Rao, "Long period fibre grating torsion sensor measuring twist rate and determining twist direction simultaneously," Electronics Letters, vol. 40, no. 3, pp. 164166, 2004.

[11] Y.-J. Rao, Y.-P. Wang, Z.-L. Ran, and T. Zhu, "Novel fiberoptic sensors based on long-period fiber gratings written by high-frequency $\mathrm{CO}_{2}$ laser pulses," Journal of Lightwave Technology, vol. 21, no. 5, pp. 1320-1327, 2003.

[12] Y. P. Wang and Y. J. Rao, " $\mathrm{CO}_{2}$-laser induced LPFG torsion characteristics depending on length of twisted fibre," Electronics Letters, vol. 40, no. 18, pp. 1101-1103, 2004.

[13] L. Shi, T. Zhu, Y. Fan, K. S. Chiang, and Y. Rao, “Torsion sensing with a fiber ring laser incorporating a pair of rotary long-period fiber gratings," Optics Communication, vol. 284, no. 22, pp. 5299-5302, 2011.

[14] X. Dong, Z. Xie, Y. Song et al., "Highly sensitive torsion sensor based on long period fiber grating fabricated by femtosecond laser pulses," Optics \& Laser Technology, vol. 97, pp. 248253, 2017.

[15] S. W. James and R. P. Tatam, "Optical fibre long-period grating sensors: characteristics and application," Measurement Science and Technology, vol. 14, no. 5, pp. R49-R61, 2003.

[16] Y. Wang, "Review of long period fiber gratings written by $\mathrm{CO}_{2}$ laser," Journal of Applied Physics, vol. 108, no. 8, article 081101, 2010. 
[17] B. L. Bachim and T. K. Gaylord, "Polarization-dependent loss and birefringence in long-period fiber gratings," Applied Optics, vol. 42, no. 34, pp. 6816-6823, 2003.

[18] J. M. P. Coelho, C. Silva, M. Nespereira, M. Abreu, and J. M. Rebordão, "Writing of long period fiber gratings using $\mathrm{CO}_{2}$ laser radiation," in Advances in Optical Fiber Technology: Fundamental Optical Phenomena and Applications, pp. 287314, InTech, 2015.

[19] M. Nespereira, D. Castro Alves, J. M. Coelho, F. Monteiro, M. Abreu, and J. M. Rebordão, "Repeatability analysis on LPFGs written by a $\mathrm{CO}_{2}$ laser," in Proceedings Volume 9286, Second International Conference on Applications of Optics and Photonics; 92863W, Aveiro, Portugal, August 2014.

[20] G. R. C. Possetti, R. C. Kamikawachi, M. Muller, and J. L. Fabris, "Metrological evaluation of optical fiber gratingbased sensors: an approach towards the standardization," Journal of Lightwave Technology, vol. 30, no. 8, pp. 10421052, 2012. 


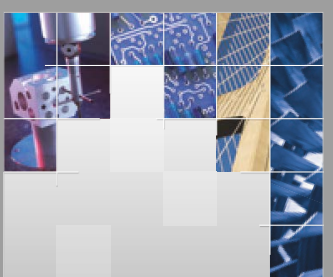

\section{Enfincering}
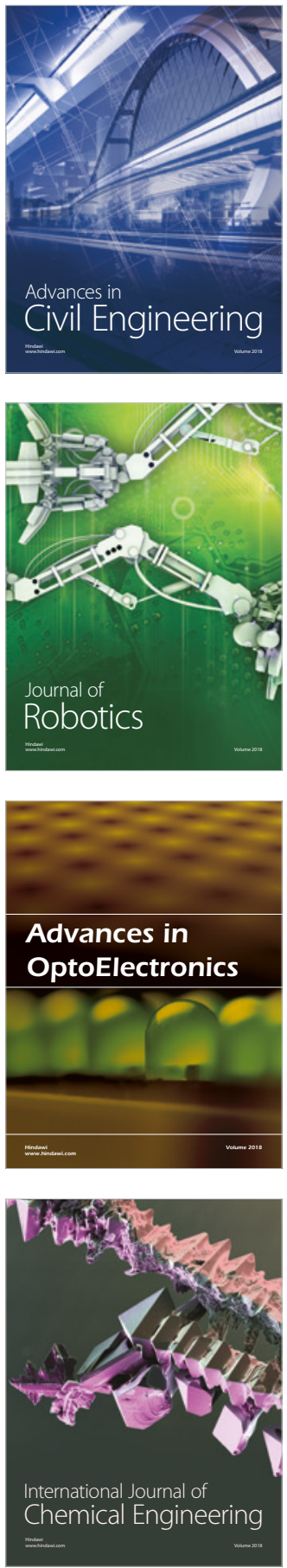

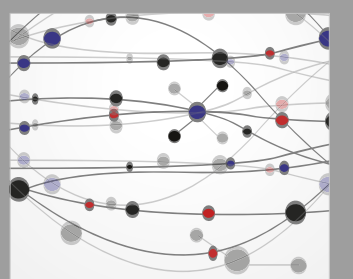

\section{Rotating \\ Machinery}

The Scientific World Journal

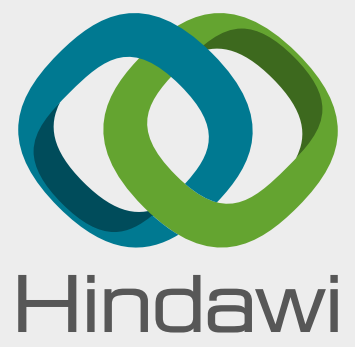

Submit your manuscripts at

www.hindawi.com
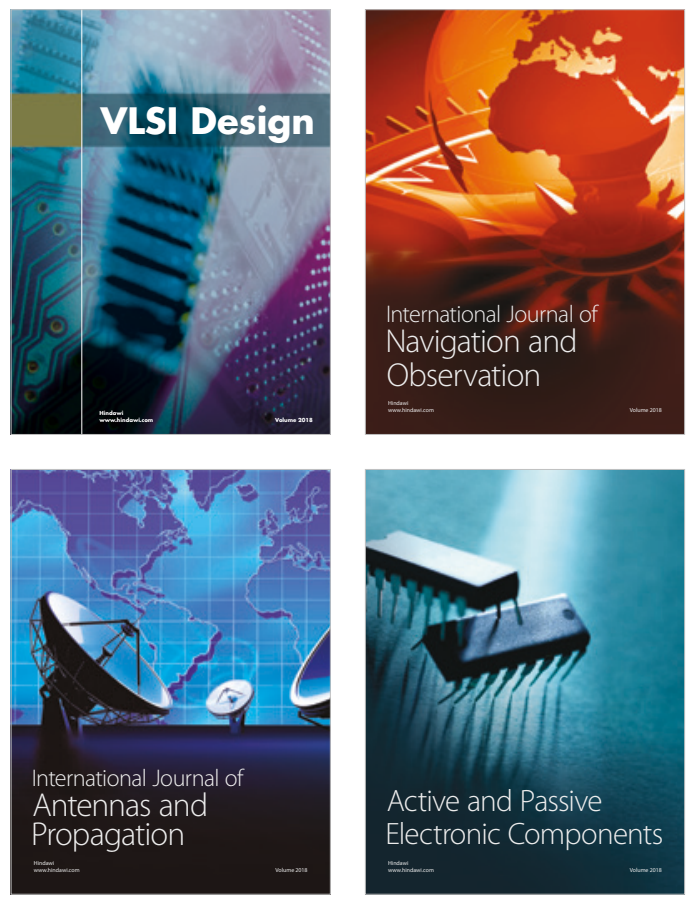
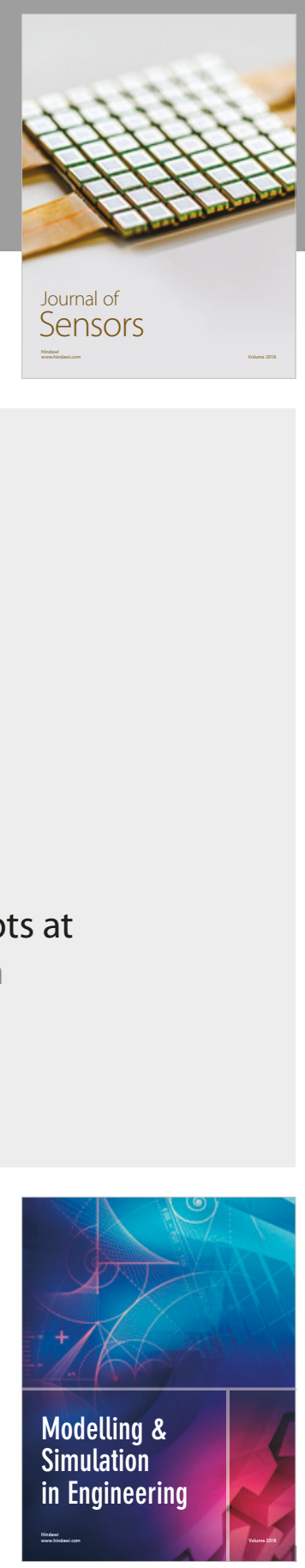

\section{Advances \\ Multimedia}
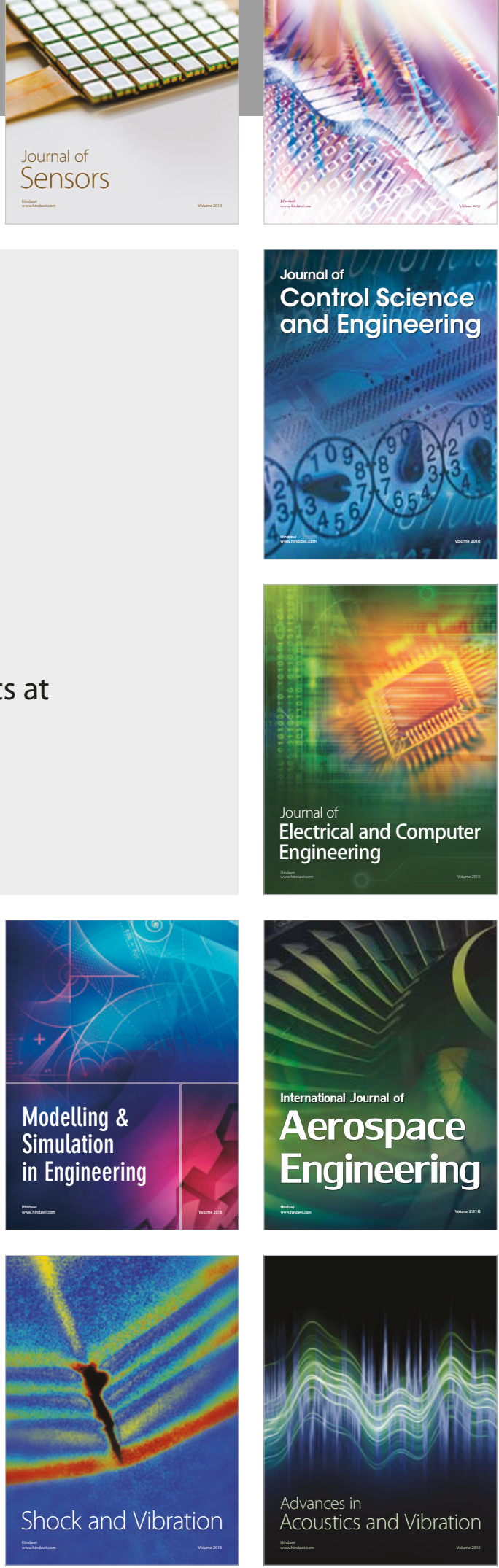